\title{
Soliton, Breather-Like and Dark-Soliton-Breather-Like Solutions for the Coupled Long-Wave-Short-Wave System
}

\section{Kuai Bi}

Taiyuan University of Technology

Hui-Qin Hao ( $\square$ math0351@sina.com )

Taiyuan University of Technology

Jian-Wen Zhang

Taiyuan University of Technology

Rui Guo

Taiyuan University of technology

\section{Research Article}

Keywords: Coupled long-wave-short-wave system, N-soliton solution, Breather-like solutions, Dark solitonbreather-like solutions, Hirota bilinear method, Asymptotic analysis method

Posted Date: December 29th, 2021

DOI: https://doi.org/10.21203/rs.3.rs-1172888/v1

License: (9) This work is licensed under a Creative Commons Attribution 4.0 International License. Read Full License

Version of Record: A version of this preprint was published at Nonlinear Dynamics on January 20th, 2022. See the published version at https://doi.org/10.1007/s11071-022-07209-9. 


\title{
Soliton, Breather-like and Dark-soliton-breather-like solutions for the coupled long-wave-short-wave system
}

\author{
Kuai Bi · Hui-Qin Hao · Jian-Wen Zhang · Rui Guo
}

Received: date / Accepted: date

\begin{abstract}
In this paper, we will obtain the exact $N$ soliton solution of the coupled long-wave-short-wave system via the developed Hirota bilinear method. Through manipulating the relevant parameters, we will construct different types of solutions which include breather-like solutions and dark-soliton-breather-like solutions. Moreover, we will demonstrate that the interactions of twosoliton and two-breather-like solutions are all elastic through asymptotic analysis method. Finally, we will display the interactions through illustrations.
\end{abstract}

PACS 05.45.Yv; 02.30.Ik; 42.81.Dp

Keywords Coupled long-wave-short-wave system . $N$-soliton solution - Breather-like solutions · Darksoliton-breather-like solutions · Hirota bilinear method · Asymptotic analysis method

\section{Introduction}

It is well-known that Davey-Stewartson system is a classical $(2+1)$ dimensional model that describes weak non-

Kuai Bi

School of Mathematics, Taiyuan University of Technology, Taiyuan 030024, China

Hui-Qin Hao

School of Mathematics, Taiyuan University of Technology, Taiyuan 030024, China

Corresponding author; E-mail: math0351@sina.com

Jian-Wen Zhang

School of Mathematics, Taiyuan University of Technology, Taiyuan 030024, China

Rui Guo

School of Mathematics, Taiyuan University of Technology, Taiyuan 030024, China linear water waves, namely

$$
\begin{aligned}
& i A_{t}+\frac{1}{2} \omega^{\prime \prime}(k) A_{z z}+\frac{c_{g}}{2 k} A_{y y}=\gamma_{1}|A|^{2} A+\gamma_{2} A \Phi_{z}, \\
& \left(g h-c_{g}^{2}\right) \Phi_{z z}+g h \Phi_{y y}=-\gamma_{3}\left(|A|^{2}\right)_{z}
\end{aligned}
$$

where $\Phi$ is the amplitude of long wave, $A$ is the complex amplitude of the short wave, $g$ is the acceleration of gravity, $h$ is the mean depth of the water, $\omega, k$ and $c_{g}$ denote the frequency, wavenumber and group velocity of the weak nonlinear water waves respectively [14]. Early literatures have supported the result that the two equations can be converted into a single equation by comparing Eqs. (1.1) and the nonlinear Schrödinger equation [3-7]. The forms of Eqs. (1.1) are completely different from that of the nonlinear Schrödinger equation, but it can be written in another way which makes the connection obvious $[3,8]$. So the equivalent forms for Eqs. (1.1) can be given as follows

$$
\begin{gathered}
i A_{t}+\lambda A_{z z}+\mu A_{y y}=\nu|A|^{2} A+\nu_{1} A Q, \\
\left(g h-c_{g}^{2}\right) Q_{z z}+g h Q_{y y}=\kappa\left(|A|^{2}\right)_{y y} .
\end{gathered}
$$

On the one hand, Eqs. (1.2) can be reduced to the nonlinear Schrödinger equation in the case where $A$ is independent of $y$ and $Q=0$. On the other hand, it should be noted that Eqs. (1.2) are invalid for the $\operatorname{case}(\nu=0)$ of long-wave resonances with short-wave $[2$, $3]$. So when the more general nonlinear Schrödinger equation break down due to a singularity of the coefficient $\nu$ of the cubic nonlinear term, Ref. [4] derives the scalar long-wave-short-wave resonance equations describing this resonance through a different analysis and scaling, the dispersion of the short wave is balanced by the nonlinear interaction of the short wave and the long 
wave, while the evolution of the long wave is driven by the self-interaction of the short wave. The scalar longwave-short-wave resonance equations can be given as follows

$i A_{t}+\lambda A_{z z}=B A$,

$B_{t}=-\alpha\left(|A|^{2}\right)_{z}$

where $B$ denotes the amplitude of long longitudinal wave, $A$ denotes the complex amplitude of the short transverse wave, $t$ and $z$ are time and space coordinates respectively $[9,10]$. Eqs. (1.3) can't describe the resonance interaction in the deep-water limit, and its travelling-wave solutions are unstable [4]. A general theory that reveals various phenomena related to physical properties such as resonances, instabilities and steady state solutions in interactions between short waves and long waves has been analyzed [11]. Hence, we consider the coupled long-wave-short-wave system which generalizes the scalar long-wave-short-wave resonance equations. It has been given as follows

$$
\left\{\begin{array}{l}
i p_{t}-2 p_{z z}+2 p(\eta-\omega)=0 \\
i q_{t}-2 q_{z z}+2 q(\eta-\omega)=0 \\
\eta_{t}-4(p \bar{q}+q \bar{p})_{z}=0
\end{array}\right.
$$

This system consists of two short-wave components and one long-wave, in which the group velocity of the transverse wave represented by two short-wave components resonates with the phase velocity of the longitudinal wave represented by a long-wave. Besides, $p$ and $q$ are complex functions representing two short-wave components, $\eta$ is a real function representing the long-wave, $\omega$ is an arbitrary real constant, $\bar{p}$ and $\bar{q}$ are complex conjugations of $p$ and $q$ respectively [12-14]. System (1.4) is integrable and has been extensively applied in various aspects $[11,12]$.

To our knowledge, many different types of solutions of System (1.4) have been obtained. For example, inclined periodic breather solutions, Ma breather solutions, Akhmediev breather solutions, rogue wave solutions and rational homoclinic solutions of System (1.4) have been obtained by Hirota two-soliton method [13, 14]. In particular, the traits of various breather solutions have been discussed which may provide us with useful information on the dynamics of the relevant physical fields. Moreover a Bäcklund transformation for planewave solutions with linear instability of System (1.4) has been obtained using the dressing method [12]. The explicit expression of the periodic orbit on the homoclinic manifold of a torus of plane waves has been constructed by evaluating the Bäcklund transformation.

However, there are still some solutions that have not been discussed for System (1.4). The purpose of this paper is mainly to generate bright soliton, dark soliton, breather-like and dark-soliton-breather-like solutions via the developed Hirota bilinear method $[15,16]$ and discuss the dynamic behaviors of two-soliton and two-breather-like solutions. Hirota bilinear method is an important tool for solving the exact solutions of nonlinear evolution equations. Once the bilinear equations of System (1.4) are obtained through certain transformation, a series of exact solutions can be obtained regularly by using the truncated parameter expansions at different levels. Asymptotic analysis method [17,18] can be used to study the dynamic behaviors of solitons before and after interactions, and related physical properties of solitons can be derived which may provide us with useful information on the dynamics of the relevant physical fields.

The structure of this paper is designed as follows. We will obtain the exact $N$-soliton solution of the coupled long-wave-short-wave system by using the developed Hirota bilinear method in Sec. 2. The breatherlike solutions will be obtained in Sec. 3 and mixed (dark-soliton-breather-like) solutions will be discussed in Sec. 4. The dynamic behaviors of two-soliton and two-breather-like solutions will be illustrated in Sec. 5 . The conclusions will be summarized in Sec. 6 .

\section{Soliton solutions for the coupled long-wave-short-wave system}

\subsection{Developed Hirota bilinear method}

Firstly, we introduce the relevant variable transformation $[19,20]$

$$
\begin{aligned}
p & =\frac{G}{F}, \\
q & =\frac{H}{F}, \\
\eta & =b_{0}-2(\ln F)_{z z} .
\end{aligned}
$$

Substituting Eqs. (2.1) into System (1.4), the coupled bilinear differential equations of System (1.4) can be obtained as

$$
\left\{\begin{array}{l}
{\left[i D_{t}-2 D_{z}^{2}+2\left(b_{0}-\omega\right)\right] G \cdot F=0,} \\
{\left[i D_{t}-2 D_{z}^{2}+2\left(b_{0}-\omega\right)\right] H \cdot F=0,} \\
\left(D_{t} D_{z}-\lambda\right) F \cdot F+4(G \bar{H}+\bar{G} H)=0,
\end{array}\right.
$$

where $G$ and $H$ are complex valued functions, $F$ is a real valued function, $b_{0}$ is a constant, and $\lambda$ is an integral constant, $\bar{G}$ and $\bar{H}$ are complex conjugates of $G$ and $H$, 
respectively. The $D$-operator is defined by

$$
\begin{aligned}
D_{t}^{n} D_{z}^{m} F(z, t) G(z, t)= & \left(\frac{\partial}{\partial t}-\frac{\partial}{\partial t^{\prime}}\right)^{n}\left(\frac{\partial}{\partial z}-\frac{\partial}{\partial z^{\prime}}\right)^{m} \\
& \times F(z, t) G\left(z^{\prime}, t^{\prime}\right) \mid z^{\prime}=z, t^{\prime}=t
\end{aligned}
$$

Next, we expand the functions $G, H$ and $F$ with the parameter $\varepsilon$ as follows

$$
\begin{aligned}
G= & m g_{0}+n \varepsilon g_{1}+m \varepsilon^{2} g_{0} g_{2}+n \varepsilon^{3} g_{3} \\
& +m \varepsilon^{4} g_{0} g_{4}+n \varepsilon^{5} g_{5}+m \varepsilon^{6} g_{0} g_{6}+\ldots, \\
H= & a g_{0}+b \varepsilon g_{1}+a \varepsilon^{2} g_{0} g_{2}+b \varepsilon^{3} g_{3} \\
& +a \varepsilon^{4} g_{0} g_{4}+b \varepsilon^{5} g_{5}+a \varepsilon^{6} g_{0} g_{6}+\ldots, \\
F=1 & +\varepsilon^{2} f_{2}+\varepsilon^{4} f_{4}+\varepsilon^{6} f_{6}+\ldots .
\end{aligned}
$$

Substituting Eqs. (2.4) into (2.2), we can obtain a set of equations by comparing the coefficients of $\varepsilon^{n}$ :

$$
\begin{aligned}
\varepsilon^{0}: 0= & D_{1} g_{0} \cdot 1, \\
0= & D_{2} 1 \cdot 1-4(m \bar{a}+a \bar{m})\left|g_{0}\right|^{2}, \\
\varepsilon^{1}: 0= & D_{1} g_{1} \cdot 1, \\
\varepsilon^{2}: 0= & D_{1}\left(g_{0} \cdot f_{2}+g_{0} g_{2} \cdot 1\right), \\
0= & D_{2}\left(f_{2} \cdot 1+1 \cdot f_{2}\right)+4\left[(m \bar{a}+a \bar{m})\left|g_{0}\right|^{2}\left(g_{2}+\bar{g}_{2}\right)\right. \\
& \left.+(n \bar{b}+b \bar{n})\left|g_{1}\right|^{2}\right] \\
\varepsilon^{3}: 0= & D_{1}\left(g_{3} \cdot 1+g_{1} \cdot f_{2}\right), \\
\varepsilon^{4}: 0= & D_{1}\left(g_{0} g_{4} \cdot 1+g_{0} g_{2} \cdot f_{2}+g_{0} \cdot f_{4}\right), \\
0= & D_{2}\left(f_{4} \cdot 1+f_{2} \cdot f_{2}+1 \cdot f_{4}\right)+4\left[(m \bar{a}+a \bar{m})\left|g_{0}\right|^{2}\right. \\
& \left.\times\left(g_{4}+\left|g_{2}\right|^{2}+\bar{g}_{4}\right)+(n \bar{b}+b \bar{n})\left(g_{3} \bar{g}_{1}+g_{1} \bar{g}_{3}\right)\right],
\end{aligned}
$$

with

$$
\begin{aligned}
& D_{1}=i D_{t}-2 D_{z}^{2}+2\left(b_{0}-\omega\right), \\
& D_{2}=D_{t} D_{z}-\lambda .
\end{aligned}
$$

In the above expressions, $\varepsilon$ is an arbitrary real constant, $m, n, a$ and $b$ are arbitrary complex constants, $\bar{m}, \bar{n}, \bar{a}$ and $\bar{b}$ are complex conjugates of $m, n, a$ and $b$, respectively. In the process of calculation, we find that the parameters satisfy the relations $m \bar{a}+a \bar{m} \neq 0$, $n \bar{b}+b \bar{n} \neq 0, m \bar{b}+a \bar{n}=0$ and $n \bar{a}+b \bar{m}=0$.

\subsection{One-soliton solutions}

In order to obtain the one-soliton solution of System (1.4), we can take

$G=m g_{0}+n \varepsilon g_{1}+m \varepsilon^{2} g_{0} g_{2}$,

$H=a g_{0}+b \varepsilon g_{1}+a \varepsilon^{2} g_{0} g_{2}$,

$F=1+\varepsilon^{2} f_{2}$.
Substituting Eqs. (2.5) into (2.2), we can obtain

$$
\begin{array}{llrl}
g_{0} & =\gamma_{0} e^{i \eta_{0}}, & g_{1} & =\alpha_{1} e^{\eta_{1}}, \\
g_{2} & =e^{\eta_{1}+\bar{\eta}_{1}+A}, & f_{2} & =e^{\eta_{1}+\bar{\eta}_{1}+\mu}, \\
\eta_{0} & =k_{0} t+p_{0} z, & k_{0} & =-2\left(\omega-b_{0}-p_{0}^{2}\right), \\
\eta_{1} & =k_{1} t+p_{1} z+\varphi_{0}, & k_{1} & =-2 i\left(\omega-b_{0}+p_{1}^{2}\right) .
\end{array}
$$

We assume $\Pi=m \bar{a}+a \bar{m}, \Theta=n \bar{b}+b \bar{n}$, then

$$
\begin{aligned}
& \lambda=4 \Pi\left|\gamma_{0}\right|^{2}, \quad e^{A}=\frac{p_{0}+i p_{1}}{p_{0}-i \bar{p}_{1}} e^{\mu} \\
& e^{\mu}=\frac{\Theta\left|\alpha_{1}\right|^{2}}{\left(p_{1}+\bar{p}_{1}\right)^{2}}\left[i\left(p_{1}-\bar{p}_{1}\right)+\frac{\Pi\left|\gamma_{0}\right|^{2}}{\left|p_{1}-i p_{0}\right|^{2}}\right]^{-1} .
\end{aligned}
$$

Thus, we obtain the one-soliton solutions as follows

$$
\begin{aligned}
& p=\frac{m \gamma_{0} e^{i \eta_{0}}\left(1+e^{\eta_{1}+\bar{\eta}_{1}+A}\right)+n \alpha_{1} e^{\eta_{1}}}{1+e^{\eta_{1}+\bar{\eta}_{1}+\mu}} \\
& q=\frac{a \gamma_{0} e^{i \eta_{0}}\left(1+e^{\eta_{1}+\bar{\eta}_{1}+A}\right)+b \alpha_{1} e^{\eta_{1}}}{1+e^{\eta_{1}+\bar{\eta}_{1}+\mu}} \\
& \eta=b_{0}-2\left(\ln \left(1+e^{\eta_{1}+\bar{\eta}_{1}+\mu}\right)\right)_{z z}
\end{aligned}
$$

In the above expressions, $p_{0}$ is an arbitrary real constant, $\gamma_{0}, \varphi_{0}, \alpha_{1}$ and $p_{1}$ are arbitrary complex constants. In this case of $\gamma_{0}=0$, the one-soliton solutions can be expressed as follows

$$
\begin{aligned}
& p=\frac{n \alpha_{1} e^{\eta_{1 I}}}{2 e^{\frac{\mu}{2}} \cosh \left(\eta_{1 R}+\frac{\mu}{2}\right)}, \\
& q=\frac{b \alpha_{1} e^{\eta_{1 I}}}{2 e^{\frac{\mu}{2}} \cosh \left(\eta_{1 R}+\frac{\mu}{2}\right)}, \\
& \eta=b_{0}-2\left(\ln \left(1+e^{2 \eta_{1 R}+\mu}\right)\right)_{z z},
\end{aligned}
$$

where $\eta_{1 R}$ and $\eta_{1 I}$ represent the real part and imaginary part of $\eta_{1}$, respectively. Especially, when $p_{1}=1+1.2 i$, we can get the bright one-soliton and dark one-soliton solutions of System (1.4) as shown in Figs. 1(a) and 1(b) respectively.

\subsection{Two-soliton solutions}

In order to obtain the two-soliton solution of System (1.4), we can take

$$
\begin{aligned}
& G=m g_{0}+n \varepsilon g_{1}+m \varepsilon^{2} g_{0} g_{2}+n \varepsilon^{3} g_{3}+m \varepsilon^{4} g_{0} g_{4}, \\
& H=a g_{0}+b \varepsilon g_{1}+a \varepsilon^{2} g_{0} g_{2}+b \varepsilon^{3} g_{3}+a \varepsilon^{4} g_{0} g_{4}, \\
& F=1+\varepsilon^{2} f_{2}+\varepsilon^{4} f_{4} .
\end{aligned}
$$

Substituting Eqs. (2.8) into (2.2), we can obtain 


$$
\begin{aligned}
G= & m \gamma_{0} e^{i \eta_{0}}\left(1+e^{\eta_{1}+\bar{\eta}_{1}+A_{11}}+e^{\eta_{1}+\bar{\eta}_{2}+A_{12}}+e^{\eta_{2}+\bar{\eta}_{1}+A_{21}}\right. \\
& \left.+e^{\eta_{2}+\bar{\eta}_{2}+A_{22}}+e^{\eta_{1}+\eta_{2}+\bar{\eta}_{1}+\bar{\eta}_{2}+\rho}\right)+n\left(\alpha_{1} e^{\eta_{1}}+\alpha_{2} e^{\eta_{2}}\right. \\
& \left.+e^{\eta_{1}+\eta_{2}+\bar{\eta}_{1}+\chi_{1}}+e^{\eta_{1}+\eta_{2}+\bar{\eta}_{2}+\chi_{2}}\right), \\
H= & a \gamma_{0} e^{i \eta_{0}}\left(1+e^{\eta_{1}+\bar{\eta}_{1}+A_{11}}+e^{\eta_{1}+\bar{\eta}_{2}+A_{12}}+e^{\eta_{2}+\bar{\eta}_{1}+A_{21}}\right. \\
& \left.+e^{\eta_{2}+\bar{\eta}_{2}+A_{22}}+e^{\eta_{1}+\eta_{2}+\bar{\eta}_{1}+\bar{\eta}_{2}+\rho}\right)+b\left(\alpha_{1} e^{\eta_{1}}+\alpha_{2} e^{\eta_{2}}\right. \\
& \left.+e^{\eta_{1}+\eta_{2}+\bar{\eta}_{1}+\chi_{1}}+e^{\eta_{1}+\eta_{2}+\bar{\eta}_{2}+\chi_{2}}\right), \\
F= & +e^{\eta_{1}+\bar{\eta}_{1}+\mu_{11}}+e^{\eta_{1}+\bar{\eta}_{2}+\mu_{12}}+e^{\eta_{2}+\bar{\eta}_{1}+\mu_{21}} \\
& +e^{\eta_{2}+\bar{\eta}_{2}+\mu_{22}}+e^{\eta_{1}+\eta_{2}+\bar{\eta}_{1}+\bar{\eta}_{2}+\Omega},
\end{aligned}
$$

the relevant variables are as follows

$$
\begin{aligned}
& \eta_{0}=k_{0} t+p_{0} z, \quad k_{0}=-2\left(\omega-b_{0}-p_{0}^{2}\right), \\
& \eta_{j}=k_{j} t+p_{j} z+\varphi_{j}, \quad k_{j}=-2 i\left(\omega-b_{0}+p_{j}^{2}\right) \text {, } \\
& \lambda=4 \Pi\left|\gamma_{0}\right|^{2}, \quad \quad e^{A_{j k}}=\frac{p_{0}+i p_{j}}{p_{0}-i \bar{p}_{k}} e^{\mu_{j k}}, \\
& e^{\chi_{1}}=\frac{\left(p_{2}-p_{1}\right)^{2}}{\Theta \bar{\alpha}_{1}}\left[i\left(p_{2}+p_{1}\right)+\frac{\Pi\left|\gamma_{0}\right|^{2}}{\left(p_{0}+i p_{2}\right)\left(p_{0}+i p_{1}\right)}\right] \\
& \times e^{\mu_{21}+\mu_{11}}, \\
& e^{\chi_{2}}=\frac{\left(p_{2}-p_{1}\right)^{2}}{\Theta \bar{\alpha}_{2}}\left[i\left(p_{2}+p_{1}\right)+\frac{\Pi\left|\gamma_{0}\right|^{2}}{\left(p_{0}+i p_{2}\right)\left(p_{0}+i p_{1}\right)}\right] \\
& \times e^{\mu_{22}+\mu_{12}}, \\
& e^{\rho}=\frac{\left(p_{0}+i p_{1}\right)\left(p_{0}+i p_{2}\right)}{\left(p_{0}-i \bar{p}_{1}\right)\left(p_{0}-i \bar{p}_{2}\right)} e^{\Omega},
\end{aligned}
$$

with

$$
\begin{aligned}
e^{\mu_{j k}}= & \frac{\Theta \alpha_{j} \bar{\alpha}_{k}}{\left(p_{j}+\bar{p}_{k}\right)^{2}}\left[i\left(p_{j}-\bar{p}_{k}\right)+\frac{\Pi\left|\gamma_{0}\right|^{2}}{\left(p_{0}+i p_{j}\right)\left(p_{0}-i \bar{p}_{k}\right)}\right]^{-1}, \\
e^{\Omega}= & \frac{\left|p_{2}-p_{1}\right|^{4}}{\Theta^{2}\left|\alpha_{1} \alpha_{2}\right|^{2}}\left|i\left(p_{2}+p_{1}\right)+\frac{\Pi\left|\gamma_{0}\right|^{2}}{\left(p_{0}+i p_{2}\right)\left(p_{0}+i p_{1}\right)}\right|^{2} \\
& \times e^{\mu_{11}+\mu_{12}+\mu_{21}+\mu_{22}} .
\end{aligned}
$$

Thus, we obtain the two-soliton solutions as follows $p=\frac{m \gamma_{0} e^{i \eta_{0}}\left(G^{1}\right)+n\left(G^{2}\right)}{F^{1}}$,

$q=\frac{a \gamma_{0} e^{i \eta_{0}}\left(G^{1}\right)+b\left(G^{2}\right)}{F^{1}}$,

$\eta=b_{0}-2\left(\ln \left(F^{1}\right)\right)_{z z}$,

with

$$
\begin{aligned}
G^{1}=1 & +e^{\eta_{1}+\bar{\eta}_{1}+A_{11}}+e^{\eta_{1}+\bar{\eta}_{2}+A_{12}}+e^{\eta_{2}+\bar{\eta}_{1}+A_{21}} \\
& +e^{\eta_{2}+\bar{\eta}_{2}+A_{22}}+e^{\eta_{1}+\eta_{2}+\bar{\eta}_{1}+\bar{\eta}_{2}+\rho}, \\
G^{2}= & \alpha_{1} e^{\eta_{1}}+\alpha_{2} e^{\eta_{2}}+e^{\eta_{1}+\eta_{2}+\bar{\eta}_{1}+\chi_{1}}+e^{\eta_{1}+\eta_{2}+\bar{\eta}_{2}+\chi_{2}}, \\
F^{1}=1 & +e^{\eta_{1}+\bar{\eta}_{1}+\mu_{11}}+e^{\eta_{1}+\bar{\eta}_{2}+\mu_{12}}+e^{\eta_{2}+\bar{\eta}_{1}+\mu_{21}} \\
& +e^{\eta_{2}+\bar{\eta}_{2}+\mu_{22}}+e^{\eta_{1}+\eta_{2}+\bar{\eta}_{1}+\bar{\eta}_{2}+\Omega} .
\end{aligned}
$$

In the above expressions, $j, k=1,2, p_{0}$ is an arbitrary real constant, $\alpha_{j}, p_{j}, \varphi_{j}(j=1,2)$ and $\gamma_{0}$ are arbitrary complex constants. Moreover, $e^{\mu_{11}}$ and $e^{\mu_{22}}$ are real constants, $e^{\mu_{12}}$ and $e^{\mu_{21}}$ are complex constants. When the parameters take some specific values, the two-soliton solutions of System (1.4) can be obtained. When $p_{1}=1.2+i, p_{2}=1.4+i$, we can get the parallel two-soliton solution as shown in Fig. 2(a). When $p_{1}=1.2+0.4 i, p_{2}=0.4+2 i$, we can obtain the intersectant two-soliton solution as shown in Fig. 2(b), and the shapes and amplitudes of the two solitons remain unchanged before and after collision. Similarly, we can obtain the parallel dark-two-soliton and the intersectant dark-two-soliton solutions as shown in Figs. 2(c) and 2(d) respectively.

\subsection{Three-soliton solutions}

In order to obtain the three-soliton solution of System (1.4), we can take

$$
\begin{aligned}
G= & m g_{0}+n \varepsilon g_{1}+m \varepsilon^{2} g_{0} g_{2}+n \varepsilon^{3} g_{3} \\
& +m \varepsilon^{4} g_{0} g_{4}+n \varepsilon^{5} g_{5}+m \varepsilon^{6} g_{0} g_{6}, \\
H= & a g_{0}+b \varepsilon g_{1}+a \varepsilon^{2} g_{0} g_{2}+b \varepsilon^{3} g_{3} \\
& +a \varepsilon^{4} g_{0} g_{4}+b \varepsilon^{5} g_{5}+a \varepsilon^{6} g_{0} g_{6}, \\
F= & 1+\varepsilon^{2} f_{2}+\varepsilon^{4} f_{4}+\varepsilon^{6} f_{6} .
\end{aligned}
$$

Substituting Eqs. (2.11) into (2.2), we can get:

$$
\begin{aligned}
& g_{0}=\gamma_{0} e^{i \eta_{0}}, \quad g_{1}=e^{\eta_{1}}+e^{\eta_{2}}+e^{\eta_{3}}, \\
& g_{2}=e^{\eta_{1}+\bar{\eta}_{1}+A_{11}}+e^{\eta_{1}+\bar{\eta}_{2}+A_{12}}+e^{\eta_{1}+\bar{\eta}_{3}+A_{13}} \\
& +e^{\eta_{2}+\bar{\eta}_{1}+A_{21}}+e^{\eta_{2}+\bar{\eta}_{2}+A_{22}}+e^{\eta_{2}+\bar{\eta}_{3}+A_{23}} \\
& +e^{\eta_{3}+\bar{\eta}_{1}+A_{31}}+e^{\eta_{3}+\bar{\eta}_{2}+A_{32}}+e^{\eta_{3}+\bar{\eta}_{3}+A_{33}}, \\
& f_{2}=e^{\eta_{1}+\bar{\eta}_{1}+\mu_{11}}+e^{\eta_{1}+\bar{\eta}_{2}+\mu_{12}}+e^{\eta_{1}+\bar{\eta}_{3}+\mu_{13}} \\
& +e^{\eta_{2}+\bar{\eta}_{1}+\mu_{21}}+e^{\eta_{2}+\bar{\eta}_{2}+\mu_{22}}+e^{\eta_{2}+\bar{\eta}_{3}+\mu_{23}} \\
& +e^{\eta_{3}+\bar{\eta}_{1}+\mu_{31}}+e^{\eta_{3}+\bar{\eta}_{2}+\mu_{32}}+e^{\eta_{3}+\bar{\eta}_{3}+\mu_{33}}, \\
& g_{3}=e^{\eta_{1}+\eta_{2}+\bar{\eta}_{1}+\chi_{124}}+e^{\eta_{1}+\eta_{2}+\bar{\eta}_{2}+\chi_{125}}+e^{\eta_{1}+\eta_{2}+\bar{\eta}_{3}+\chi_{126}} \\
& +e^{\eta_{1}+\eta_{3}+\bar{\eta}_{1}+\chi_{134}}+e^{\eta_{1}+\eta_{3}+\bar{\eta}_{2}+\chi_{135}}+e^{\eta_{1}+\eta_{3}+\bar{\eta}_{3}+\chi_{136}} \\
& +e^{\eta_{2}+\eta_{3}+\bar{\eta}_{1}+\chi_{234}}+e^{\eta_{2}+\eta_{3}+\bar{\eta}_{2}+\chi_{235}}+e^{\eta_{2}+\eta_{3}+\bar{\eta}_{3}+\chi_{236}}, \\
& g_{4}=e^{\eta_{1}+\eta_{2}+\bar{\eta}_{1}+\bar{\eta}_{2}+\rho_{1245}}+e^{\eta_{1}+\eta_{3}+\bar{\eta}_{1}+\bar{\eta}_{2}+\rho_{1345}} \\
& +e^{\eta_{1}+\eta_{2}+\bar{\eta}_{1}+\bar{\eta}_{3}+\rho_{1246}}+e^{\eta_{1}+\eta_{3}+\bar{\eta}_{1}+\bar{\eta}_{3}+\rho_{1346}} \\
& +e^{\eta_{1}+\eta_{2}+\bar{\eta}_{2}+\bar{\eta}_{3}+\rho_{1256}}+e^{\eta_{1}+\eta_{3}+\bar{\eta}_{2}+\bar{\eta}_{3}+\rho_{1356}} \\
& +e^{\eta_{2}+\eta_{3}+\bar{\eta}_{1}+\bar{\eta}_{2}+\rho_{2345}}+e^{\eta_{2}+\eta_{3}+\bar{\eta}_{1}+\bar{\eta}_{3}+\rho_{2346}} \\
& +e^{\eta_{2}+\eta_{3}+\bar{\eta}_{2}+\bar{\eta}_{3}+\rho_{2356}} \\
& f_{4}=e^{\eta_{1}+\eta_{2}+\bar{\eta}_{1}+\bar{\eta}_{2}+\Omega_{1245}}+e^{\eta_{1}+\eta_{3}+\bar{\eta}_{1}+\bar{\eta}_{2}+\Omega_{1345}} \\
& +e^{\eta_{1}+\eta_{2}+\bar{\eta}_{1}+\bar{\eta}_{3}+\Omega_{1246}}+e^{\eta_{1}+\eta_{3}+\bar{\eta}_{1}+\bar{\eta}_{3}+\Omega_{1346}} \\
& +e^{\eta_{1}+\eta_{2}+\bar{\eta}_{2}+\bar{\eta}_{3}+\Omega_{1256}}+e^{\eta_{1}+\eta_{3}+\bar{\eta}_{2}+\bar{\eta}_{3}+\Omega_{1356}} \\
& +e^{\eta_{2}+\eta_{3}+\bar{\eta}_{1}+\bar{\eta}_{2}+\Omega_{2345}}+e^{\eta_{2}+\eta_{3}+\bar{\eta}_{1}+\bar{\eta}_{3}+\Omega_{2346}} \\
& +e^{\eta_{2}+\eta_{3}+\bar{\eta}_{2}+\bar{\eta}_{3}+\Omega_{2356}},
\end{aligned}
$$


$g_{5}=e^{\eta_{1}+\eta_{2}+\eta_{3}+\bar{\eta}_{1}+\bar{\eta}_{2}+\delta_{45}}+e^{\eta_{1}+\eta_{2}+\eta_{3}+\bar{\eta}_{1}+\bar{\eta}_{3}+\delta_{46}}$

$$
+e^{\eta_{1}+\eta_{2}+\eta_{3}+\bar{\eta}_{2}+\bar{\eta}_{3}+\delta_{56}}
$$

$g_{6}=e^{\eta_{1}+\eta_{2}+\eta_{3}+\bar{\eta}_{1}+\bar{\eta}_{2}+\bar{\eta}_{3}+\varpi}$

$f_{6}=e^{\eta_{1}+\eta_{2}+\eta_{3}+\bar{\eta}_{1}+\bar{\eta}_{2}+\bar{\eta}_{3}+\vartheta}$,

in which

$$
\begin{aligned}
& \eta_{0}=k_{0} t+p_{0} z \\
& k_{0}=-2\left(\omega-b_{0}-p_{0}^{2}\right), \\
& \eta_{j}=k_{j} t+p_{j} z+\varphi_{j} \\
& k_{j}=-2 i\left(\omega-b_{0}+p_{j}^{2}\right) \text {, } \\
& \lambda=4 \Pi\left|\gamma_{0}\right|^{2}, \\
& e^{\chi_{j_{1} j_{2} j_{3}}}=\frac{\left(p_{j_{2}}-p_{j_{1}}\right)^{2}}{\Theta} \\
& \times\left[i\left(p_{j_{2}}+p_{j_{1}}\right)+\frac{\Pi\left|\gamma_{0}\right|^{2}}{\left(p_{0}+i p_{j_{2}}\right)\left(p_{0}+i p_{j_{1}}\right)}\right] \\
& \times e^{\mu_{j_{1} j_{3}-3} \mu_{j_{2} j_{3}-3}}, \\
& e^{\rho_{j_{1} j_{2} j_{3} j_{4}}}=\frac{\left(p_{0}+i p_{j_{1}}\right)\left(p_{0}+i p_{j_{2}}\right)}{\left(p_{0}-i \bar{p}_{j_{3}-3}\right)\left(p_{0}-i \bar{p}_{j_{4}-3}\right)} e^{\Omega_{j_{1} j_{2} j_{3} j_{4}}} \\
& e^{\delta_{j_{3} j_{4}}}=\frac{\left(p_{1}-p_{2}\right)^{2}\left(p_{1}-p_{3}\right)^{2}\left(p_{2}-p_{3}\right)^{2}\left(p_{j_{3}-3}-p_{j_{4}-3}\right)^{2}}{\Theta^{4}} \\
& \times\left[i\left(p_{1}+p_{2}\right)+\frac{\Pi\left|\gamma_{0}\right|^{2}}{\left(p_{0}+i p_{2}\right)\left(p_{0}+i p_{1}\right)}\right] \\
& \times\left[i\left(p_{1}+p_{3}\right)+\frac{\Pi\left|\gamma_{0}\right|^{2}}{\left(p_{0}+i p_{3}\right)\left(p_{0}+i p_{1}\right)}\right] \\
& \times\left[i\left(p_{2}+p_{3}\right)+\frac{\Pi\left|\gamma_{0}\right|^{2}}{\left(p_{0}+i p_{3}\right)\left(p_{0}+i p_{2}\right)}\right] \\
& \times\left[-i\left(\bar{p}_{j_{3}-3}+\bar{p}_{j_{4}-3}\right)\right. \\
& \left.+\frac{\Pi\left|\gamma_{0}\right|^{2}}{\left(p_{0}-i \bar{p}_{j_{3}-3}\right)\left(p_{0}-i \bar{p}_{j_{4}-3}\right)}\right] \\
& \times e^{\mu_{1 j_{3}-3} \mu_{2 j_{3}-3} \mu_{3 j_{3}-3} \mu_{1 j_{4}-3} \mu_{2 j_{4}-3} \mu_{3 j_{4}-3}}, \\
& e^{\varpi}=\frac{\left(p_{0}+i p_{1}\right)\left(p_{0}+i p_{2}\right)\left(p_{0}+i p_{3}\right)}{\left(p_{0}-i \bar{p}_{1}\right)\left(p_{0}-i \bar{p}_{2}\right)\left(p_{0}-i \bar{p}_{3}\right)} e^{\vartheta},
\end{aligned}
$$

with

$$
\begin{aligned}
& e^{\mu_{j k}}=\frac{\Theta}{\left(p_{j}+\bar{p}_{k}\right)^{2}}\left[i\left(p_{j}-\bar{p}_{k}\right)+\frac{\Pi\left|\gamma_{0}\right|^{2}}{\left(p_{0}+i p_{j}\right)\left(p_{0}-i \bar{p}_{k}\right)}\right]^{-1}, \\
& e^{\Omega_{j_{1} j_{2} j_{3} j_{4}}=} \frac{\left(p_{j_{1}}-p_{j_{2}}\right)^{2}\left(\bar{p}_{j_{3}-3}-\bar{p}_{j_{4}-3}\right)^{2}}{\Theta^{2}} \\
& \times\left[i\left(p_{j_{1}}+p_{j_{2}}\right)+\frac{\Pi\left|\gamma_{0}\right|^{2}}{\left(p_{0}+i p_{j_{2}}\right)\left(p_{0}+i p_{j_{1}}\right)}\right] \\
& \times\left[-i\left(\bar{p}_{j_{3}-3}+\bar{p}_{j_{4}-3}\right)\right. \\
&\left.+\frac{\Pi\left|\gamma_{0}\right|^{2}}{\left(p_{0}-i \bar{p}_{j_{3}-3}\right)\left(p_{0}-i \bar{p}_{j_{4}-3}\right)}\right] \\
& \times e^{\mu_{j_{1} j_{3}-3} \mu_{j_{2} j_{3}-3} \mu_{j_{1} j_{4}-3} \mu_{j_{2} j_{4}-3}},
\end{aligned}
$$

$$
\begin{aligned}
e^{\vartheta}= & \frac{\left|p_{1}-p_{2}\right|^{4}\left|p_{1}-p_{3}\right|^{4}\left|p_{2}-p_{3}\right|^{4}}{\Theta^{6}} \\
& \times\left|i\left(p_{1}+p_{2}\right)+\frac{\Pi\left|\gamma_{0}\right|^{2}}{\left(p_{0}+i p_{2}\right)\left(p_{0}+i p_{1}\right)}\right|^{2} \\
& \times\left|i\left(p_{1}+p_{3}\right)+\frac{\Pi\left|\gamma_{0}\right|^{2}}{\left(p_{0}+i p_{3}\right)\left(p_{0}+i p_{1}\right)}\right|^{2} \\
& \times\left|i\left(p_{2}+p_{3}\right)+\frac{\Pi\left|\gamma_{0}\right|^{2}}{\left(p_{0}+i p_{3}\right)\left(p_{0}+i p_{2}\right)}\right|^{2} \\
& \times e^{\mu_{11}+\mu_{21}+\mu_{31}+\mu_{12}+\mu_{22}+\mu_{32}+\mu_{13}+\mu_{23}+\mu_{33}} .
\end{aligned}
$$

Thus, we obtain the three-soliton solutions as follows

$$
\begin{aligned}
& p=\frac{m \gamma_{0} e^{i \eta_{0}}\left(G_{1}\right)+n\left(G_{2}\right)}{F_{1}}, \\
& q=\frac{a \gamma_{0} e^{i \eta_{0}}\left(G_{1}\right)+b\left(G_{2}\right)}{F_{1}}, \\
& \eta=b_{0}-2\left(\ln \left(F_{1}\right)\right)_{z z},
\end{aligned}
$$

with

$$
\begin{aligned}
G_{1}= & +\sum e^{\eta_{j_{1}}+\bar{\eta}_{j_{3}-3}+A_{j_{1} j_{3}}}+e^{\eta_{1}+\eta_{2}+\eta_{3}+\bar{\eta}_{1}+\bar{\eta}_{2}+\bar{\eta}_{3}+\varpi} \\
& +\sum e^{\eta_{j_{1}}+\eta_{j_{2}}+\bar{\eta}_{j_{3}-3}+\bar{\eta}_{j_{4}-3}+\rho_{j_{1} j_{2} j_{3} j_{4}}} \\
G_{2}= & e^{\eta_{1}}+e^{\eta_{2}}+e^{\eta_{3}}+\sum e^{\eta_{j_{1}}+\eta_{j_{2}}+\bar{\eta}_{j_{3}-3}+\chi_{j_{1} j_{2} j_{3}}} \\
& +\sum e^{\eta_{1}+\eta_{2}+\eta_{3}+\bar{\eta}_{j_{3}-3}+\bar{\eta}_{j_{4}-3}+\delta_{j_{3} j_{4}}}, \\
F_{1}= & +\sum e^{\eta_{j_{1}}+\bar{\eta}_{j_{3}-3}+\mu_{j_{1} j_{3}}}+e^{\eta_{1}+\eta_{2}+\eta_{3}+\bar{\eta}_{1}+\bar{\eta}_{2}+\bar{\eta}_{3}+\vartheta} \\
& +\sum e^{\eta_{j_{1}}+\eta_{j_{2}}+\bar{\eta}_{j_{3}-3}+\bar{\eta}_{j_{4}-3}+\Omega_{j_{1} j_{2} j_{3} j_{4}}} .
\end{aligned}
$$

In the above expressions, $j, k, j_{1}, j_{2}=1,2,3, j_{3}, j_{4}=$ $4,5,6, p_{0}$ is an arbitrary real constant, $p_{j}, \varphi_{j}(j=1,2,3)$ and $\gamma_{0}$ are arbitrary complex constants. Besides, $e^{\mu_{j k}}(j=k)$ and $e^{\Omega_{j_{1} j_{2} j_{3} j_{4}}}\left(j_{3}-3=j_{1}, j_{4}-3=j_{2}\right)$ are real constants, $e^{\mu_{j k}}(j \neq k)$ and $e^{\Omega_{j_{1} j_{2} j_{3} j_{4}}}\left(j_{3}-3 \neq j_{1}\right.$ or $\left.j_{4}-3 \neq j_{2}\right)$ are complex constants. When the parameters take some specific values, the three-soliton solutions of System (1.4) can be obtained. When $p_{1}=1.2+i, p_{2}=1.3+i$, $p_{3}=1.4+i$, the parallel three-soliton solution can be obtained as shown in Fig. 3(a). When $p_{1}=1+1.9 i$, $p_{2}=1.5+0.5 i, p_{3}=0.8+1.9 i$, the three-soliton solution can be obtained as shown in Fig. 3(b), in which one soliton intersects with the other two solitons. When $p_{1}=1+1.5 i, p_{2}=1.5+0.5 i, p_{3}=1.8+0.7 i$, the intersectant three-soliton solution can be obtained as shown in Fig. 3(c). Similarly, we can get the corresponding darkthree-soliton solutions as shown in Figs. 3(d), 3(e) and $3(\mathrm{f})$.

\subsection{N-soliton solutions}


Proceeding further, $G, H$ and $F$ in $N$-soliton solution can be expressed as follows

$$
\begin{aligned}
& G=m \gamma_{0} e^{i \eta_{0}}\left[\sum_{\beta=0,1} \Gamma_{1}(\beta) \exp \left(\sum_{j=1}^{2 N} \beta_{j} \eta_{j}+\sum_{1 \leq i<j}^{2 N} \beta_{i} \beta_{j} A_{i j}\right)\right] p=\frac{m \gamma_{0} e^{i \eta_{0}}\left[\sum_{\beta=0,1} \Gamma_{1}(\beta) \exp \left(\sum_{j=1}^{2 N} \beta_{j} \eta_{j}+\sum_{1 \leq i<j}^{2 N} \beta_{i} \beta_{j} A_{i j}\right)\right]}{\sum_{\beta=0,1} \Gamma_{1}(\beta) \exp \left(\sum_{j=1}^{2 N} \beta_{j} \eta_{j}+\sum_{1 \leq i<j}^{2 N} \beta_{i} \beta_{j} \mu_{i j}\right)} \\
& +n \sum_{\beta=0,1} \Gamma_{2}(\beta) \exp \left(\sum_{j=1}^{2 N} \beta_{j} \eta_{j}+\sum_{1 \leq i<j}^{2 N} \beta_{i} \beta_{j} \mu_{i j}\right) \text {, } \\
& H=a \gamma_{0} e^{i \eta_{0}}\left[\sum_{\beta=0,1} \Gamma_{1}(\beta) \exp \left(\sum_{j=1}^{2 N} \beta_{j} \eta_{j}+\sum_{1 \leq i<j}^{2 N} \beta_{i} \beta_{j} A_{i j}\right)\right] q=\frac{a \gamma_{0} e^{i \eta_{0}}\left[\sum_{\beta=0,1} \Gamma_{1}(\beta) \exp \left(\sum_{j=1}^{2 N} \beta_{j} \eta_{j}+\sum_{1 \leq i<j}^{2 N} \beta_{i} \beta_{j} A_{i j}\right)\right]}{\sum_{\beta=0,1} \Gamma_{1}(\beta) \exp \left(\sum_{j=1}^{2 N} \beta_{j} \eta_{j}+\sum_{1 \leq i<j}^{2 N} \beta_{i} \beta_{j} \mu_{i j}\right)} \\
& +b \sum_{\beta=0,1} \Gamma_{2}(\beta) \exp \left(\sum_{j=1}^{2 N} \beta_{j} \eta_{j}+\sum_{1 \leq i<j}^{2 N} \beta_{i} \beta_{j} \mu_{i j}\right) \text {, } \\
& F=\sum_{\beta=0,1} \Gamma_{1}(\beta) \exp \left(\sum_{j=1}^{2 N} \beta_{j} \eta_{j}+\sum_{1 \leq i<j}^{2 N} \beta_{i} \beta_{j} \mu_{i j}\right), \\
& +\frac{b \sum_{\beta=0,1} \Gamma_{2}(\beta) \exp \left(\sum_{j=1}^{2 N} \beta_{j} \eta_{j}+\sum_{1 \leq i<j}^{2 N} \beta_{i} \beta_{j} \mu_{i j}\right)}{\sum_{\beta=0,1} \Gamma_{1}(\beta) \exp \left(\sum_{j=1}^{2 N} \beta_{j} \eta_{j}+\sum_{1 \leq i<j}^{2 N} \beta_{i} \beta_{j} \mu_{i j}\right)}, \\
& \eta=b_{0}-2\left(\ln \left(\sum_{\beta=0,1} \Gamma_{1}(\beta) \exp \left(\sum_{j=1}^{2 N} \beta_{j} \eta_{j}+\sum_{1 \leq i<j}^{2 N} \beta_{i} \beta_{j} \mu_{i j}\right)\right)\right)_{z z}
\end{aligned}
$$

with

$$
\begin{array}{lr}
\eta_{0}=k_{0} t+p_{0} z, & k_{0}=-2\left(\omega-b_{0}-p_{0}^{2}\right), \\
\eta_{j}=k_{j} t+p_{j} z+\varphi_{j}, & k_{j}=-2 i\left(\omega-b_{0}+p_{j}^{2}\right), \\
\eta_{j+N}=\bar{\eta}_{j}, \quad p_{j+N}=\bar{p}_{j}, \quad \lambda=4 \Pi\left|\gamma_{0}\right|^{2},
\end{array}
$$$$
\Gamma_{1}(\beta)= \begin{cases}1, & \sum_{j=1}^{N} \beta_{j+N}=\sum_{j=1}^{N} \beta_{j}, \\ 0, & \sum_{j=1}^{N} \beta_{j+N} \neq \sum_{j=1}^{N} \beta_{j},\end{cases}
$$$$
\Gamma_{2}(\beta)= \begin{cases}1, & 1+\sum_{j=1}^{N} \beta_{j+N}=\sum_{j=1}^{N} \beta_{j}, \\ 0, & 1+\sum_{j=1}^{N} \beta_{j+N} \neq \sum_{j=1}^{N} \beta_{j},\end{cases}
$$

and

$$
\begin{gathered}
e^{A_{i j}}=\left\{\begin{array}{c}
\frac{p_{0}+i p_{i}}{p_{0}-i p_{j}} e^{\mu_{i j}} \\
i=1,2, \ldots, N, j=N+1, \ldots, 2 N, \\
\frac{1}{\left(p_{0}+i p_{i}\right)\left(p_{0}+i p_{j}\right)} e^{\mu_{i j}} \\
i=1,2, \ldots, N, j=1,2, \ldots, N, \\
\left(p_{0}-i p_{i}\right)\left(p_{0}-i p_{j}\right) e^{\mu_{i j}} \\
i=N+1, \ldots, 2 N, j=N+1, \ldots, 2 N,
\end{array}\right. \\
e^{\mu_{i j}}=\left\{\begin{array}{c}
\frac{\Theta}{\left(p_{i}+p_{j}\right)^{2}}\left[i\left(p_{i}-p_{j}\right)+\frac{\Pi\left|\gamma_{0}\right|^{2}}{\left(p_{0}+i p_{i}\right)\left(p_{0}-i p_{j}\right)}\right]^{-1} \\
i=1,2, \ldots, N, j=N+1, \ldots, 2 N, \\
\frac{\left(p_{i}-p_{j}\right)^{2}}{\Theta}\left[i\left(p_{i}+p_{j}\right)+\frac{\Pi\left|\gamma_{0}\right|^{2}}{\left(p_{0}+i p_{i}\right)\left(p_{0}+i p_{j}\right)}\right] \\
i=1,2, \ldots, N, j=1,2, \ldots, N, \\
\frac{\left(p_{i}-p_{j}\right)^{2}}{\Theta}\left[i\left(p_{i}+p_{j}\right)+\frac{\Pi\left|\gamma_{0}\right|^{2}}{\left(p_{0}-i p_{i}\right)\left(p_{0}-i p_{j}\right)}\right] \\
i=N+1, \ldots, 2 N, j=N+1, \ldots, 2 N .
\end{array}\right.
\end{gathered}
$$

In the above expressions, $p_{0}$ is an arbitrary real constant, $p_{j}, \varphi_{j}(j=1,2,3, \ldots, N)$ and $\gamma_{0}$ are arbitrary complex constants, $\sum_{\beta=0,1}$ is the summation of all possible combinations of $\beta_{i}, \beta_{j}=0,1, \sum_{1 \leq i<j}^{2 N}$ is the summation of all possible combinations where $i$ and $j$ satisfy $1 \leq i<j \leq 2 N$.

\section{Breather-like solutions for the coupled long-wave-short-wave system}

In this part, we consider the case of $\gamma_{0} \neq 0$. Because $p$ and $q$ have similar properties, we only consider the results obtained by $p$.

\subsection{One-breather-like solutions}

In the case of $\gamma_{0} \neq 0$, Eqs. (2.6) can be rewritten as follows

$$
\begin{aligned}
p= & \frac{1}{2} m \gamma_{0} e^{i \eta_{0}}\left[1+\kappa-(1-\kappa) \tanh \left(\eta_{1 R}+\frac{\mu}{2}\right)\right] \\
& +\frac{1}{2} n \alpha_{1} \frac{e^{i \eta_{1 I}}}{e^{\frac{\mu}{2}} \cosh \left(\eta_{1 R}+\frac{\mu}{2}\right)}, \\
q= & \frac{1}{2} a \gamma_{0} e^{i \eta_{0}}\left[1+\kappa-(1-\kappa) \tanh \left(\eta_{1 R}+\frac{\mu}{2}\right)\right] \\
& +\frac{1}{2} b \alpha_{1} \frac{e^{i \eta_{1 I}}}{e^{\frac{\mu}{2}} \cosh \left(\eta_{1 R}+\frac{\mu}{2}\right)}, \\
\eta= & b_{0}-2\left(\ln \left(1+e^{2 \eta_{1 R}+\mu}\right)\right)_{z z},
\end{aligned}
$$

with

$\kappa=\frac{p_{0}+i p_{1}}{p_{0}-i \bar{p}_{1}}$, 
where $\eta_{1 R}$ and $\eta_{1 I}$ represent the real part and imaginary part of $\eta_{1}$, respectively. Therefore, when $\gamma_{0}=1$, $p_{1}=1+1.2 i$, the one-breather-like solution can be obtained as shown in Fig. 4(a). We can see such obvious breather behavior as peaks and valleys. Compared with Fig. 4(a), when the imaginary part of $p_{1}$ decreases, the propagative direction of one-breather-like in Fig. 4(b) changes. When the real part of $p_{1}$ decreases, it can be found that the amplitude of one-breather-like in Fig. 4(c) diminishes. In Fig. 4(d), we can find that the initial position of one-breather-like changes with the changes of $\varphi_{0}$.

\subsection{Two-breather-like solutions}

Here, we consider the case of $\gamma_{0}=1$ in Eqs. (2.10). When $p_{1}=1.2+i, p_{2}=1.4+i$, the parallel twobreather-like solution can be obtained as shown in Fig. 5(a) When the real part of $p_{2}$ or $p_{1}$ decrease, the amplitude of corresponding breather-like reduce as shown in Figs. 5(b), 5(c) and 5(d), respectively.

Next, we consider the case of $\gamma_{0}=0.3$ in Eqs. (2.10). When $p_{1}=0.4+i, p_{2}=1+0.1 i$, the intersectant two-breather-like solution can be obtained as shown in Fig. 6(a). Compared with Fig. 6(a), when the real part of $p_{1}$ increases, it can be found that the amplitude of corresponding breather-like increases in Fig. 6(b). In Fig. 6(c), when the imaginary part of $p_{1}$ increases, the angle between the two breather-like augments. Finally, it can be found that the initial position of one of the two breather-like changes when $\varphi_{1}$ changes by comparing Figs. 6(a) and 6(d).

\subsection{Three-breather-like solutions}

Similarly, the three-breather-like solutions can be obtained as shown in Figs. 7. It can be seen that the initial position of one of the three breather-like changes when $\varphi_{3}$ changes by comparing Fig. $7(\mathrm{a})$ with $7(\mathrm{~b})$. Compared with Fig. $7(\mathrm{a})$, when real parts of $p_{1}, p_{2}$ and $p_{3}$ all decrease, it can be found that the amplitudes of three breather-like all reduce in Fig. 7(c). What's more, Fig. $7(\mathrm{~d})$ shows the three-breather-like solution that one breather-like intersects with the other two breather-like.

\section{Dark-soliton-breather-like solutions for the coupled long-wave-short-wave system}

In this part, we consider the case of $\gamma_{0}=1$ and $p_{0}=$ -0.7 . Because $p$ and $q$ have similar properties, we only consider the results obtained by $p$.

\subsection{Dark-soliton-one-breather-like solutions}

Here, we consider the case of $\gamma_{0}=1, p_{0}=-0.7$ in Eqs. (2.10). When $p_{1}=0.6+i, p_{2}=0.75+0.5 i$, the dark-soliton-one-breather-like solution can be obtained as shown in Fig. 8(a). Compared with Fig. 8(a), when the real part of $p_{1}$ reduces, it can be seen that the amplitude of breather-like decreases in Fig. 8(b). In Fig. 8(c), when the imaginary part of $p_{1}$ increases, it can be found that the propagative direction of the breather-like changes. Finally, Fig. 8(d) shows that the dark-soliton-one-breather-like solution can degenerate into one-breather-like solution when the values of $p_{1}$ and $p_{2}$ are both $0.6+i$.

\subsection{Dark-soliton-two-breather-like solution}

Next, we consider the case of $\gamma_{0}=1$ and $p_{0}=-0.7$ in Eqs. (2.12). In this case, we can obtain the darksoliton-two-breather-like solution as shown in Fig. 9(a). When the values of $p_{1}$ and $p_{3}$ are both $0.7+i$, the dark-soliton-two-breather-like solution can degrade into dark-soliton-one-breather-like solution in Fig. 9(b).

\section{Asymptotic analysis}

In this part, we consider the case of $\alpha_{1}=1, \alpha_{2}=1$ in Eqs. (2.10).

Case 1: $\gamma_{0} \neq 0$.

(i) Before collision $(t \rightarrow-\infty)$

(a) $\left(\eta_{1 R} \approx 0, \eta_{2 R} \rightarrow-\infty\right)$ :

$$
\begin{aligned}
p \rightarrow S_{1}^{-}= & \frac{m \gamma_{0} e^{i \eta_{0}}\left(1+e^{2 \eta_{1 R}+A_{11}}\right)+n e^{\eta_{1}}}{1+e^{2 \eta_{1 R}+\mu_{11}}} \\
= & \frac{1}{2} m \gamma_{0} e^{i \eta_{0}}\left[1+\kappa_{1}-\left(1-\kappa_{1}\right) \tan \left(\eta_{1 R}+\frac{\mu_{11}}{2}\right)\right] \\
& +n \Lambda_{1} e^{i \eta_{1 I}} \operatorname{sech}\left(\eta_{1 R}+\frac{\mu_{11}}{2}\right)
\end{aligned}
$$

with

$$
\kappa_{1}=\frac{p_{0}+i p_{1}}{p_{0}-i \bar{p}_{1}}, \Lambda_{1}=\frac{1}{2} \exp \left(-\frac{\mu_{11}}{2}\right) .
$$

(b) $\left(\eta_{2 R} \approx 0, \eta_{1 R} \rightarrow-\infty\right):$

$$
\begin{aligned}
p \rightarrow S_{2}^{-}= & \frac{m \gamma_{0} e^{i \eta_{0}}\left(1+e^{2 \eta_{2 R}+A_{22}}\right)+n e^{\eta_{2}}}{1+e^{2 \eta_{1 R}+\mu_{11}}} \\
= & \frac{1}{2} m \gamma_{0} e^{i \eta_{0}}\left[1+\kappa_{2}-\left(1-\kappa_{2}\right) \tan \left(\eta_{2 R}+\frac{\mu_{22}}{2}\right)\right] \\
& +n \Lambda_{2} e^{i \eta_{2 I}} \operatorname{sech}\left(\eta_{2 R}+\frac{\mu_{22}}{2}\right)
\end{aligned}
$$


with

$$
\kappa_{2}=\frac{p_{0}+i p_{2}}{p_{0}-i \bar{p}_{2}}, \Lambda_{2}=\frac{1}{2} \exp \left(-\frac{\mu_{22}}{2}\right) .
$$

(ii) After collision $(t \rightarrow \infty)$

(a) $\left(\eta_{1 R} \approx 0, \eta_{2 R} \rightarrow \infty\right)$ :

$$
\begin{aligned}
p \rightarrow S_{1}^{+}= & \frac{m \gamma_{0} e^{i \eta_{0}}\left(e^{A_{22}}+e^{2 \eta_{1 R}+\rho}\right)+n e^{\eta_{1}+\chi_{2}}}{e^{\mu_{22}}+e^{2 \eta_{1 R}+\Omega}} \\
= & \frac{1}{2} m \gamma_{0} e^{i \eta_{0}} \kappa_{2}\left[1+\kappa_{1}-\left(1-\kappa_{1}\right) \tanh \left(\eta_{1 R}\right.\right. \\
& \left.\left.+\frac{\Omega-\mu_{22}}{2}\right)\right]+n \Lambda_{3} e^{i \eta_{1 I}} \operatorname{sech}\left(\eta_{1 R}\right. \\
& \left.+\frac{\Omega-\mu_{22}}{2}\right)
\end{aligned}
$$

with

$$
\Lambda_{3}=\frac{1}{2} \exp \left(\chi_{2}-\frac{\Omega+\mu_{22}}{2}\right) .
$$

(b) $\left(\eta_{2 R} \approx 0, \eta_{1 R} \rightarrow \infty\right)$ :

$$
\begin{aligned}
p \rightarrow S_{2}^{+}= & \frac{m \gamma_{0} e^{i \eta_{0}}\left(e^{A_{11}}+e^{2 \eta_{2 R}+\rho}\right)+n e^{\eta_{2}+\chi_{1}}}{e^{\mu_{11}}+e^{2 \eta_{2 R}+\Omega}} \\
= & \frac{1}{2} m \gamma_{0} e^{i \eta_{0}} \kappa_{1}\left[1+\kappa_{2}-\left(1-\kappa_{2}\right) \tanh \left(\eta_{2 R}\right.\right. \\
& \left.\left.+\frac{\Omega-\mu_{11}}{2}\right)\right]+n \Lambda_{4} e^{i \eta_{2 I}} \operatorname{sech}\left(\eta_{2 R}\right. \\
& \left.+\frac{\Omega-\mu_{11}}{2}\right)
\end{aligned}
$$

with

$$
\Lambda_{4}=\frac{1}{2} \exp \left(\chi_{1}-\frac{\Omega+\mu_{11}}{2}\right)
$$

Here, we notice that $\left|\kappa_{1}\right|=\left|\kappa_{2}\right|=1,\left|\Lambda_{1}\right|^{2}=\left|\Lambda_{3}\right|^{2}$, $\left|\Lambda_{2}\right|^{2}=\left|\Lambda_{4}\right|^{2}$. That is to say, the amplitude of each breather-like in the two-breather-like solutions remains unchanged before and after the collision. The corresponding visual illustrations are shown in Fig. 6.

Table 1. Physical quantities of solitons $S_{1}$ and $S_{2}$ before and after the interaction for $p$ :

(a) $\left(\eta_{1 R} \approx 0, \eta_{2 R} \rightarrow-\infty\right)$ :

$$
p \rightarrow S_{1}^{-}=\frac{n e^{\eta_{1}}}{1+e^{2 \eta_{1} R^{+\mu_{11}}}}=n \Lambda_{1} e^{i \eta_{1 I}} \operatorname{sech}\left(\eta_{1 R}+\frac{\mu_{11}}{2}\right),
$$

(b) $\left(\eta_{2 R} \approx 0, \eta_{1 R} \rightarrow-\infty\right)$ :

$$
p \rightarrow S_{2}^{-}=\frac{n e^{\eta_{2}}}{1+e^{2 \eta_{1} R^{+\mu_{11}}}}=n \Lambda_{2} e^{i \eta_{2 I}} \operatorname{sech}\left(\eta_{2 R}+\frac{\mu_{22}}{2}\right),
$$

(ii) After collision $(t \rightarrow \infty)$

(a) $\left(\eta_{1 R} \approx 0, \eta_{2 R} \rightarrow \infty\right)$ :

$$
\begin{aligned}
p \rightarrow S_{1}^{+} & =\frac{n e^{\eta_{1}+\chi_{2}}}{e^{\mu 22}+e^{2 \eta_{1 R}+\Omega}} \\
& =n \Lambda_{3} e^{i \eta_{1 I}} \operatorname{sech}\left(\eta_{1 R}+\frac{\Omega-\mu_{22}}{2}\right), \\
(\mathrm{b})\left(\eta_{2 R} \approx\right. & \left.0, \eta_{1 R} \rightarrow \infty\right): \\
p \rightarrow S_{2}^{+} & =\frac{n e^{\eta_{2}+\chi_{1}}}{e^{\mu_{11}+e^{2 \eta_{2 R}+\Omega}}} \\
& =n \Lambda_{4} e^{i \eta_{2 I}} \operatorname{sech}\left(\eta_{2 R}+\frac{\Omega-\mu_{11}}{2}\right) .
\end{aligned}
$$

The relevant physical quantities for function $p$ before and after collision are listed in Table 1 . Here, we notice that $\left|\Lambda_{1}\right|^{2}=\left|\Lambda_{3}\right|^{2},\left|\Lambda_{2}\right|^{2}=\left|\Lambda_{4}\right|^{2}$. Therefore, Table 1 show that the collision of two solitons in the two-soliton solutions is elastic. The corresponding vi-

\begin{tabular}{|c|c|c|c|c|}
\hline Solitons & Velocities & Widths & Amplitudes & Initial phases \\
\hline$S_{1}^{-}$ & $\left|2 i\left(p_{1}+\bar{p}_{1}\right)\right|$ & $\frac{1}{\left|p_{1}+\bar{p}_{1}\right|}$ & $\left|n \Lambda_{1}\right|$ & $\frac{\mu_{11}}{2\left(p_{1}+\bar{p}_{1}\right)}$ \\
\hline$S_{2}^{-}$ & $\left|2 i\left(p_{2}+\bar{p}_{2}\right)\right|$ & $\frac{1}{\left|p_{2}+\bar{p}_{2}\right|}$ & $\left|n \Lambda_{2}\right|$ & $\frac{\mu_{22}}{2\left(p_{2}+\bar{p}_{2}\right)}$ \\
\hline$S_{1}^{+}$ & $\left|2 i\left(p_{1}+\bar{p}_{1}\right)\right|$ & $\frac{1}{\left|p_{1}+\bar{p}_{1}\right|}$ & $\left|n \Lambda_{3}\right|$ & $\frac{\Omega-\mu_{22}}{2\left(p_{1}+\bar{p}_{1}\right)}$ \\
\hline$S_{2}^{+}$ & $\left|2 i\left(p_{2}+\bar{p}_{2}\right)\right|$ & $\frac{1}{\left|p_{2}+\bar{p}_{2}\right|}$ & $\left|n \Lambda_{4}\right|$ & $\frac{\Omega-\mu_{11}}{2\left(p_{2}+\bar{p}_{2}\right)}$ \\
\hline
\end{tabular}
sual illustration as shown in Fig. 2(b).

\section{Conclusions}

In this paper, we have obtained various types of solutions of the coupled long-wave-short-wave system through the developed Hirota bilinear method. Variations of relevant parameters determine different types of solutions. In the case of $\gamma_{0}=0$, we have obtained the exact $N$ soliton solution. In the case of $\gamma_{0} \neq 0$, we have derived the breather-like solutions and studied the influence of $p_{j}$ and $\varphi_{j}$ for the transmission of the breather-like. In particular, we have obtained the dark-soliton-breatherlike solutions in the case of $\gamma_{0}=1, p_{0}=-0.7$. Finally, we have demonstrated that the interactions of two-soliton and two-breather-like solutions are all elastic through asymptotic analysis method.

\section{Acknowledgements}

We express our sincere thanks to each member of

Case 2. $\gamma_{0}=0$.

(i) Before collision $(t \rightarrow-\infty)$ our discussion group for their suggestions. This work has been supported by the National Natural Science Foundation of China under Grant No. 11905155, and 
the Shanxi Province Science Foundation for Youths, China under Grant Nos. 201801D221023 and 201801D121

\section{Conflict of interest}

The authors declare no conflicts of interest.

\section{Data availability}

The datasets generated during or analysed during the current study are available from the corresponding author on reasonable request.

\section{References}

1. Ablowitz, M.J., Segur, H.:On the evolution of packets of water waves. J. Fluid Mech. 92, 691-715 (1979).

2. Babaoglu, C.:Long-wave short-wave resonance case for a generalized Davey-Stewartson system. Chaos, Solit. \& Fract. 38, 48-54 (2008).

3. Davey, A., Stewartson, K.:On three-dimensional packets of surface waves. Proc. R. Soc. Lond. A: Math. Phys. Sci. 338, 101-110 (1974).

4. Djordjevic, V.D., Redekopp, L.G.:On two-dimensional packets of capillary-gravity waves. J. Fluid Mech. 79, 703-714 (1977).

5. Song, L.Min., Yang, Z.J., Pang, Z.G., Li, X.L., Zhang, S.M.:Interaction theory of mirror-symmetry soliton pairs in nonlocal nonlinear Schrodinger equation. Appl. Math. Lett. 90, 42-48 (2019).

6. Song, L.Min., Yang, Z.J., Li, X.L., Zhang, S.M.:Controllable Gaussian-shaped soliton clusters in strongly nonlocal media. Opt. Express 26, 1918219198 (2018)

7. Song, L.Min., Yang, Z.J., Zhang, S.M., Li, X.L.:Spiraling anomalous vortex beam arrays in strongly nonlocal nonlinear media. Phys. Rev. A 99, 063817 (2019).

8. Hasimoto, H., Ono, H.:Nonlinear modulation of gravity waves. J. Phys. Soc. Jpn. 33, 805-811 (1972).

9. Chan, H.N., Ding, E., Kedziora, D.J., Grimshaw, R., Chow, K.W.:Rogue waves for a long wave-short wave resonance model with multiple short waves. Nonlinear dyn. 85, 1-15 (2016).

10. Zhai, Y., Geng, X., Xue, B.:Riemann theta function solutions to the coupled long wave-short wave resonance equations. Anal. Math. Phys. 56, 1-26 (2020).

11. Benney, D.J.:A general theory for interactions between short and long waves. Stud. Appl. Math. 56, 81-94 (1977).

12. Wright III, O.C.:On a homoclinic manifold of a coupled long-wave-short-wave system. Commun. Nonlinear Sci. Numer. Simulat. 15, 2066-2072 (2010).

13. Wang, C., Dai, Z.:Various breathers and rogue waves for the coupled long-wave-short-wave system. Adv. Differ. Equ. 2014, 1-10 (2014).

14. Chen, W., Chen, H., Dai, Z.:Rational homoclinic solution and rogue wave solution for the coupled long-wave-shortwave system. Pramana 86, 713-717 (2016).

15. Wang, X.M., Zhang, L.L., Hu, X.X.:Various types of vector solitons for the coupled nonlinear Schrödinger equations in the asymmetric fiber couplers. Optik 219, 164989 (2020).

16. Wang, X.M., Zhang, L.L.:The nonautonomous N-soliton solutions for coupled nonlinear Schrödinger equation with arbitrary time-dependent potential. Nonlinear dyn. 88, 2291-2302 (2017).

17. Guo, R., Hao, H.Q.:Breathers and multi-soliton solutions for the higher-order generalized nonlinear Schrödinger equation. Commun. Nonlinear Sci. Numer. Simulat. 18, 2426-2435 (2013).

18. Hao, H.Q., Zhang, J.W.:Integrability aspects and soliton solutions for the inhomogeneous reduced Maxwell-Bloch system in nonlinear optics with symbolic computation. Commun. Nonlinear Sci. Numer. Simulat. 22, 1350-1359 (2015).

19. Tajiri, M., Takeuchi, K., Arai, T.:Asynchronous development of the Benjamin-Feir unstable mode: Solution of the Davey-Stewartson equation. Phys. Rev. E 64, 056622 (2001).

20. Tajiri, M., Arai, T.:Quasi-line soliton interactions of the Davey-Stewartson I equation: on the existence of longrange interaction between two quasi-line solitons through a periodic soliton. J. Phys. A: Math. Theor. 44, 235204 (2011).

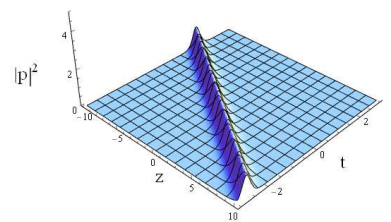

(a)

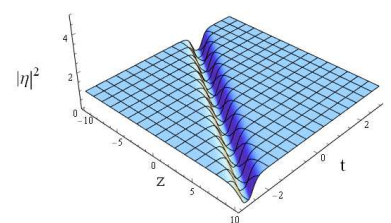

(b)
Fig. 1. One-soliton solutions for the coupled long-wave-short-wave system, the parameters are: $\varepsilon=1$, $\gamma_{0}=0, p_{0}=0, \alpha_{1}=2, b_{0}=1, \omega=1, \varphi_{0}=3, a=1, b=1$, $m=1, n=-1, p_{1}=1+1.2 i$.

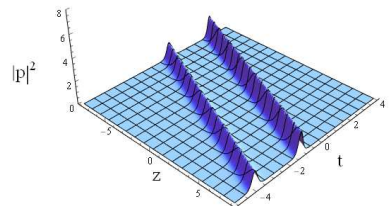

(a)

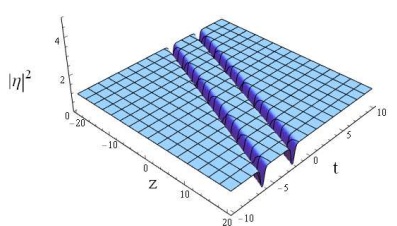

(c)

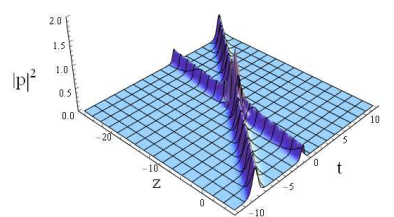

(b)

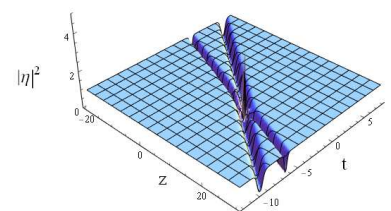

(d)
Fig. 2. Two-soliton solutions for the coupled long-wave-short-wave system, the parameters are: $\varepsilon=1$, $\gamma_{0}=0, p_{0}=0, \alpha_{1}=1, \alpha_{2}=1, b_{0}=1, \omega=1, \varphi_{1}=10$, $\varphi_{2}=1, a=1, b=1, m=1, n=-1$ and (a) $p_{1}=1.2+i$, $p_{2}=1.4+i ;$ (b) $p_{1}=1.2+0.4 i, p_{2}=0.4+2 i$; (c) $p_{1}=1+1.2 i, p_{2}=1+1.21 i ;\left(\right.$ d) $p_{1}=1+1.5 i, p_{2}=1+0.8 i$.

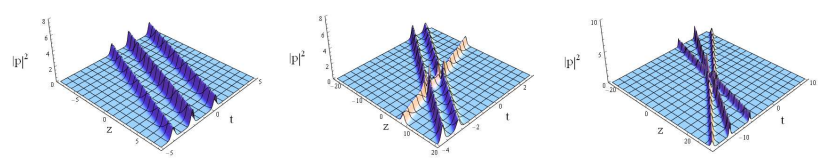


(a)

(b)

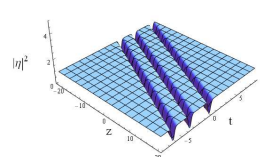

(d)

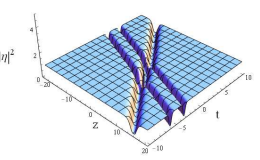

(e) (c)

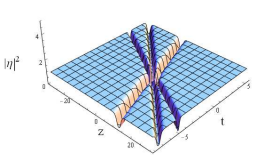

(f)

Fig. 3. Three-soliton solutions for the the coupled long-wave-short-wave system, the parameters are: $\varepsilon=1$, $\gamma_{0}=0, p_{0}=0, b_{0}=1, \omega=1, \varphi_{1}=10, \varphi_{2}=1, \varphi_{3}=6$, $a=1, b=1, m=1, n=-1$ and (a) $p_{1}=1.2+i$, $p_{2}=1.3+i, p_{3}=1.4+i$; (b) $p_{1}=1+1.9 i, p_{2}=1.5+0.5 i$, $p_{3}=0.8+1.9 i ;$ (c) $p_{1}=1+1.5 i, p_{2}=1.5+0.5 i$, $p_{3}=1.8+0.7 i ;(\mathrm{d}) p_{1}=1+1.2 i, p_{2}=1+1.21 i$, $p_{3}=1+1.22 i(\mathrm{e}) p_{1}=1+1.2 i, p_{2}=1+0.4 i, p_{3}=1+1.22 i$; (f) $p_{1}=1+1.9 i, p_{2}=1+0.4 i, p_{3}=1+1.15 i$.

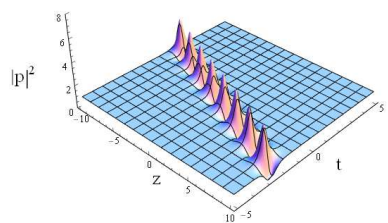

(a)

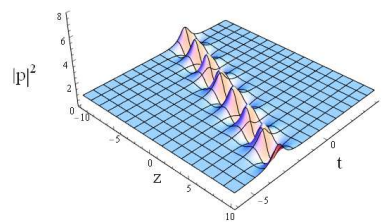

(c)

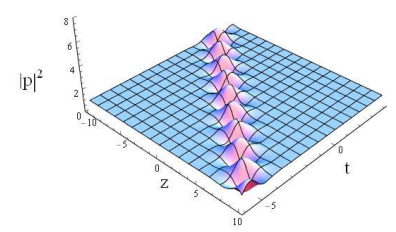

(b)

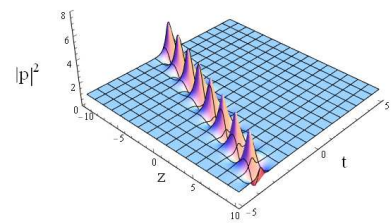

(d)

Fig. 4. One-breather-like solutions for the coupled long-wave-short-wave system, the parameters are: $\varepsilon=1$, $\gamma_{0}=1, \alpha_{1}=2, b_{0}=1, \omega=1, p_{0}=-1, a=1, b=1, m=1$, $n=-1$ and (a) $p_{1}=1+1.2 i, \varphi_{0}=3$; (b) $p_{1}=1+0.6 i$, $\varphi_{0}=3$; (c) $p_{1}=0.6+1.2 i, \varphi_{0}=3$; (d) $p_{1}=1+1.2 i, \varphi_{0}=8$.

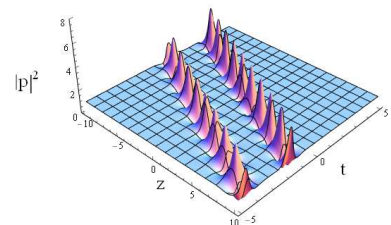

(a)

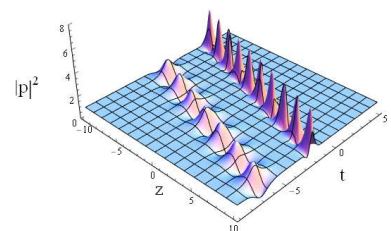

(c)

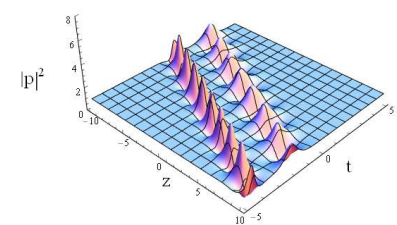

(b)

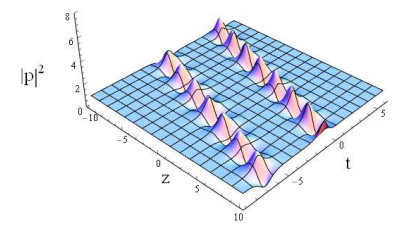

(d)

Fig. 5. Two-breather-like solutions for the coupled long-wave-short-wave system, the parameters are: $\varepsilon=1$, $\gamma_{0}=1, p_{0}=-1, \alpha_{1}=2, \alpha_{2}=1, b_{0}=1, \omega=1, \varphi_{1}=10$,

$\varphi_{2}=1, a=1, b=1, m=1, n=-1$ and (a) $p_{1}=1.2+i$, $p_{2}=1.4+i$; (b) $p_{1}=1.2+i, p_{2}=0.7+i$; (c) $p_{1}=0.6+i$, $p_{2}=1.4+i ;\left(\right.$ d) $p_{1}=0.6+i, p_{2}=0.7+i$.

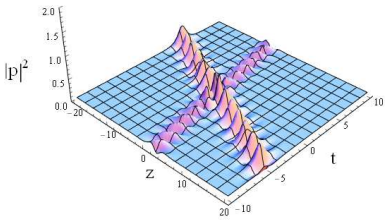

(a)

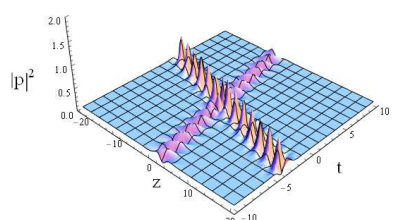

(c)

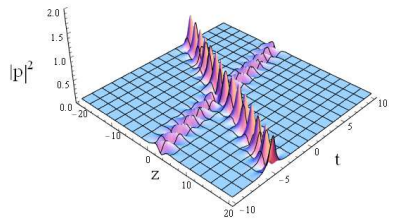

(b)

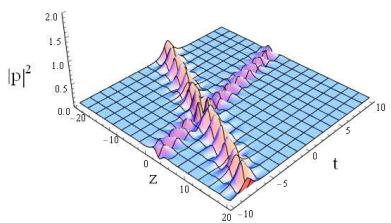

(d)
Fig. 6. Two-breather-like solutions for the coupled long-wave-short-wave system, the parameters are: $\varepsilon=1$, $\gamma_{0}=0.3, p_{0}=-1, \alpha_{1}=1, \alpha_{2}=1, b_{0}=1, \omega=1, \varphi_{2}=1$, $a=1, b=1, m=1, n=-1$ and (a) $\varphi_{1}=1, p_{1}=0.4+i$, $p_{2}=1+0.1 i$; (b) $\varphi_{1}=1, p_{1}=0.6+i, p_{2}=1+0.1 i$; (c) $\varphi_{1}=1, p_{1}=0.4+1.5 i, p_{2}=1+0.1 i ;$ (d) $\varphi_{1}=5$, $p_{1}=0.4+i, p_{2}=1+0.1 i$.

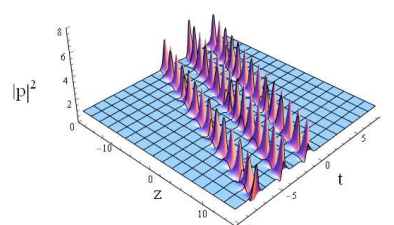

(a)

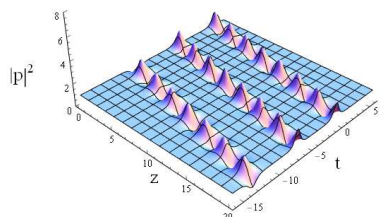

(c)

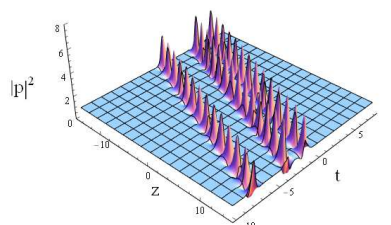

(b)

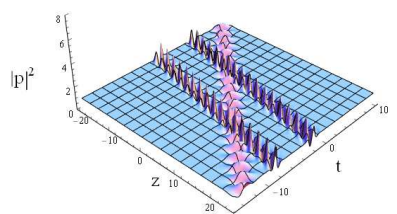

(d)
Fig. 7. Three-breather-like solutions for the coupled long-wave-short-wave system, the parameters are: $\varepsilon=1$, $\gamma_{0}=1, p_{0}=-1, b_{0}=1, \omega=1, \varphi_{1}=20, \varphi_{2}=1, a=1$, $b=1, m=1, n=-1$ and (a) $\varphi_{3}=10, p_{1}=1.2+i$, $p_{2}=1.3+i, p_{3}=1.4+i$; (b) $\varphi_{3}=10, p_{1}=0.6+i$, $p_{2}=0.65+i, p_{3}=0.7+i$; (c) $\varphi_{3}=1, p_{1}=0.5+1.9 i$, $p_{2}=0.75+0.5 i, p_{3}=0.4+1.9 i$.

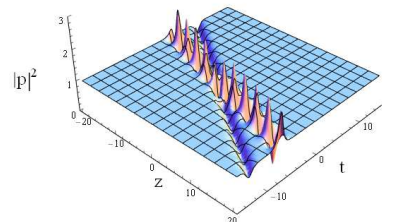

(a)

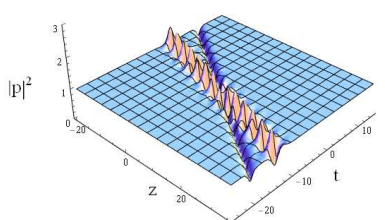

(b) 


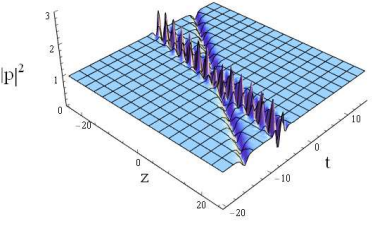

(c)

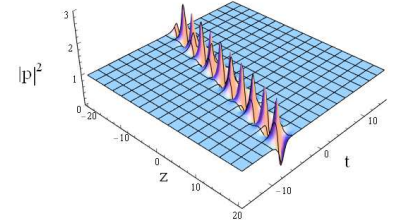

(d)

Fig. 8. Dark-soliton-one-breather-like solutions for the coupled long-wave-short-wave system, the parameters are: $\varepsilon=1, \gamma_{0}=1, p_{0}=-0.7, \alpha_{1}=2, \alpha_{2}=1, b_{0}=1, \omega=1$, $\varphi_{1}=5, \varphi_{2}=1, a=1, b=1, m=1, n=-1$, and (a) $p_{1}=0.6+i, p_{2}=0.75+0.5 i$; (b) $p_{1}=0.3+i$, $p_{2}=0.75+0.5 i ;$ (c) $p_{1}=0.3+1.5 i, p_{2}=0.75+0.5 i ;(\mathrm{d})$ $p_{1}=0.6+i, p_{2}=0.6+i$.

$|\mathrm{P}|^{2}$

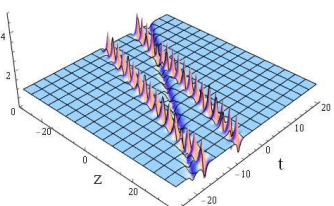

(a)

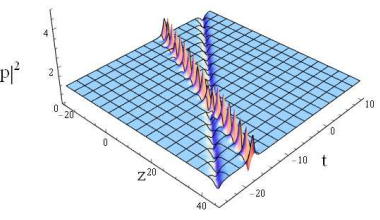

(b)

Fig. 9. Dark-soliton-two-breather-like solutions for the coupled long-wave-short-wave system, the parameters are: $\varepsilon=1, \gamma_{0}=1, p_{0}=-0.7, b_{0}=1, \omega=1, \varphi_{1}=20, \varphi_{2}=1$, $\varphi_{3}=1, a=1, b=1, m=1, n=-1$ and (a) $p_{1}=0.6+i$, $p_{2}=0.75+0.5 i, p_{3}=0.7+i$; (b) $p_{1}=0.7+i$, $p_{2}=0.75+0.5 i, p_{3}=0.7+i$. 